\title{
Strategies for Improving the Quality of Polling Service in Wireless Metropolitan Area Network
}

\author{
Zhi-Jun Yang ${ }^{1,2}$, Yang Su ${ }^{1, *}$, Hong-Wei Ding ${ }^{1}$ and Yang-Yang Ding ${ }^{1}$ \\ ${ }^{1}$ School of Information Science and Engineering, Yunnan University, Kunming, 650091, China. \\ ${ }^{2}$ The Academy for Educational Science Research, Educational Department of Yunnan Province, Kunming, 650223,China.
}

\begin{abstract}
Four kinds of service types are defined in IEEE 802.16. In order to provide the Quality of Service (Qos) for different services, the system must use a reasonable resource allocation method and scheduling algorithm to efficiently and fairly allocate bandwidth resources. Although in the IEEE 802.16 MAC, for the uplink real-time polling service (rtPS) and non-real-time polling service (nrtPS) business type of data transmission are used to polling, but do not provide business-based services. In this paper, the distinction between priority service of rtPS and nrtPS is carried out, and the simulation experiment is used to analyze the performance characteristics of the protocol under the distinction of high and low priority. Wherein the theoretical values of the average delay and the information packets are compared with the experimental values. The average query cycle and throughput are also evaluated. Which proves the validity of the improved service strategy and improves the service characteristics of the system.
\end{abstract}

\section{Introduction}

In the fields of industrial control, computer time division multiplexing, communication system protocol and computer network protocol, the control mode of polling system has been widely used because of its fairness and usability. The analysis and research of the polling system are also developing continuously. With the deepening of the research, the application of the polling system has been further expanded. The strategy of the polling service can be divided into three categories: exhaustive service, gated service and limited-k service. In practice, prioritybased services have a wide range of needs, so the distinction priority service is very necessary. In the broadband network, it is necessary to provide end-to-end service quality assurance for different types of service flows to satisfy the requirements of the traffic flow for broadband, delay jitter and packet loss rate. IEEE802.16 ${ }^{[1,2]}$, also known as the IEEE Wireless MAN air interface standard, it regulates the underlying standards (including the physical layer and the media access control layer) of the wireless access system in the 2-66 GHz frequency range, and analyzes the system coexistence background, gives the system design, configuration and frequency use scheme.

With the rapid growth of wireless data services and multimedia applications, providing QoS support in the systembusiness has become a basic requirement that must be met. The MAC protocol in IEEE 802.16 defines four types of services, specifying different QoS parameters and basic bandwidth allocation mechanisms for them. However, the standard does not specify the principles of resource allocation and scheduling between services and similar services. Therefore, how to make effective use of system resources and ensure that different QoS requirements of various services become important and challenging research work. In this paper, we focus on the scheduling problem of real-time polling service (rtPS) non-real-time polling service (nrtPS) in 802.16, and study

"Corresponding author: $\underline{\mathrm{sy} 064615 @ 163 . c o m}$

(C) The Authors, published by EDP Sciences. This is an open access article distributed under the terms of the Creative Commons Attribution License 4.0 (http://creativecommons.org/licenses/by/4.0/). 
the resource scheduling strategy between two kinds of services. At the same time,we propose a service strategy that prioritizes the two businesses. Among them, the highpriority service will use the exhaustive service operation mechanism, and low priority will use the gated service way. What's more, this kind of mixed service strategy can ensure the QoS demand and the system bandwidth allocation fairness.

\section{The development of IEEE802.16 series standards}

IEEE802.16 standard committee in July 1999 began to discuss the global unified wireless broadband metropolitan area network access standards, so far has been established including 802.16, 802.16a, 802.16c, 802.16d, 802.16e, 802.16f, etc. and a series of standards. According to the different frequency band, 802.16 system can divide into two kinds of line of sight and non line of sight, in which the 2-11 GHz band system applies to the non line of sight range, and the $10-66 \mathrm{GHz}$ band system is used in line of sight range. According to whether to support mobile features, IEEE 802. 16 standard series can also divide into fixed broadband wireless access air interface standard and mobile broadband wireless access air interface standard. So, the 802.16, 802.16a, 802.16d are fixed wireless access air interface standard, the $802.16 \mathrm{e}$ belongs to the mobile broadband wireless access air interface standard. As the current standard 802.16j, there is a backward compatibility for the earlier established IEEE802.16 standards. It uses multi-hop relay technology as the core, using a relay-based network architecture, can achieve high coverage at low cost. The technical fields corresponding to the IEEE 802. 16 part of the series of standards are shown in Table 1 below.

Table 1.The technical fields corresponding to the 802.16 part of the series of standards

\begin{tabular}{|c|c|}
\hline $\begin{array}{c}\text { Standard } \\
\text { number }\end{array}$ & Corresponding technical areas \\
\hline 802.16 & $\begin{array}{r}10-66 \mathrm{GHz} \text { fixed broadband wireless } \\
\text { access system air interface }\end{array}$ \\
\hline $802.16 \mathrm{a}$ & $\begin{array}{r}2-11 \mathrm{GHz} \text { fixed broadband access system } \\
\text { air interface }\end{array}$ \\
\hline $802.16 \mathrm{c}$ & $10-66 \mathrm{GHz}$ Compatibility of fixed \\
\hline
\end{tabular}

\begin{tabular}{|c|c|}
\hline & broadband access system \\
\hline $802.16 \mathrm{~d}$ & $\begin{array}{c}2-66 \mathrm{GHz} \text { fixed broadband access system } \\
\text { air interface }\end{array}$ \\
\hline $802.16 \mathrm{e}$ & $\begin{array}{c}2-11 \mathrm{GHz} \text { fixed and mobile broadband } \\
\text { wireless access system air interface } \\
\text { management information base }\end{array}$ \\
\hline $802.16 \mathrm{f}$ & $\begin{array}{c}2-11 \mathrm{GHz} \text { fixed broadband wireless access } \\
\text { system air interface management } \\
\text { information base (MIB) requirements }\end{array}$ \\
\hline
\end{tabular}

\section{IEEE 802.16 MAC protocol analysis}

IEEE 802.16 broadband wireless metropolitan area network consists of base station (BS) and Subscriber Station (SS). The BS is the central station, which is responsible for all communication between the network and the network. SS connects all types of users and the BS through the air interface. IEEE 802.16 MAC layer specification uses a hierarchical structure, divides into three sub-layers:

(1) Convergence Sub layer (CS), responsible for highlevel interface, convergence of the upper different business. IEEE 802.16 MAC is connection-oriented, and the protocol defines two CS sub layers to map the upper layer business as a connection. The ATM CS sub layer provides service support for ATM; Packet CS provides mapping of packet-based services such as IEEE 802.3, 802.1Q, and IP. The Packet CS sub layer defines the concept of a classifier. The classifier is a set of mapping standards. Each packet enters the network is mapped to a connection according to the rules defined by the classifier. Each connection consists of a unique connection identifier (CID) of 16 bits, according to the service quality requirements, they associate with the appropriate connection to ensure that different business QoS.

(2) Common Part Sub layer (CPS), which implements the main MAC function, is responsible for SS initialization and entry network, physical layer support and channel information management, connection and service flow management, radio resource management, data processing and retransmis sion, etc.. It supports Point to Multi-Point (PMP) network topology, downlink only BS to send, uplink broadband with multiple SS sharing, BS is responsible for uplink and downlink broadband resource allocation. The uplink adopts way that is composed of time-division multiple access (TDMA) and 
Demand Assigned Multiple Access (DAMA), and the downlink mainly adopts Time-Division Multiplex (TDM) mode. As shown in Figure 1 below: shows the uplink and downlink transmission of data.

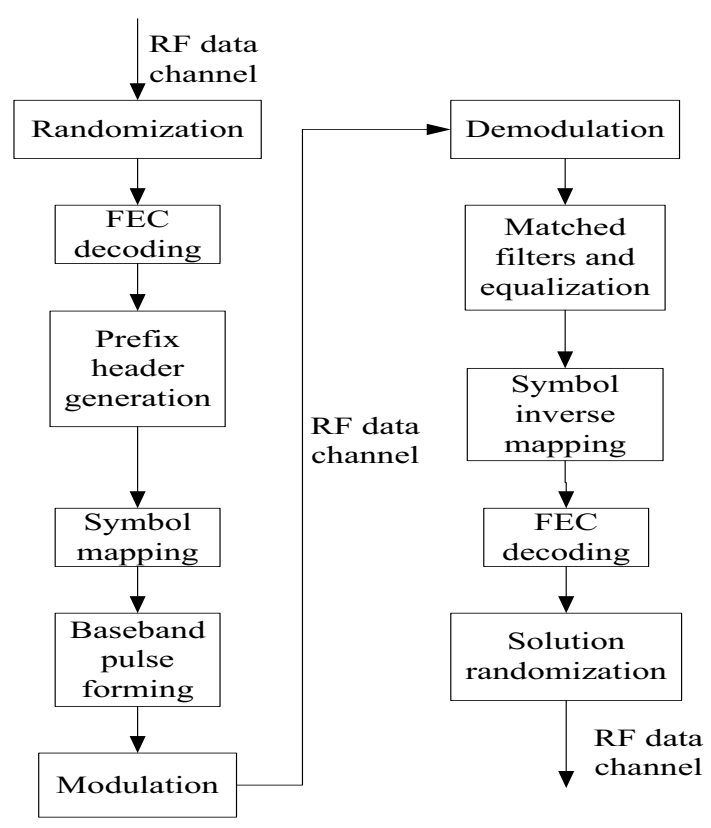

Fig.1. The uplink and downlink transmission of data.

(3) Safety Sub layer (SS), responsible for authentication and encryption.

IEEE 802.16 MAC layer implementation of the principle of QoS ${ }^{[3]}$ is to map the MAC packet transmission to the business flow, and map to the connection that has the CID logo. It can be based on the QoS parameters of the business scheduling to guarantee the MAC QoS characteristic. According to the different features and requirements of the business, IEEE 802.16 MAC in the uplink direction defines four different levels of busines $\mathrm{s}$ scheduling service type:

1) Unsolicited Grant Service (UGS) are used to support real-time business needs for fixed bandwidth, such as E1, T1, VIP, and so on.

2) Real-Time Polling Service (rtPS), rtPS is mainly used to meet the variable bandwidth real-time business needs, such as MPEG video stream.

3) Non-Real-Time Polling Service (nrtPS), nrtPS applies to the data size variable non-real-time business flow, with a certain cycle of polling connection.

4) Best Effort Service (BE), BE according to the network status for the general business to provide the greatest possible business, but it cannot guarantee QoS.

\section{Traffic service strategy based on service priority for wireless metropolitan area networks}

\subsection{Protocol model analysis}

As shown in Figure 2, the wireless computer network consists of $\mathrm{N}$ sites, each site's business is divided into two types of priority, $\lambda_{i 1}$ for the high priority service arrival rate, under the condition that the arrival process is Poisson process, the arrival process probability generation function is $A_{h}(z)$, where the mean value and the variance are $\lambda_{i 1}(i=1,2, \ldots, N)=\lambda_{h}=A_{h}^{\prime}(1)$ and $\sigma_{\lambda h}^{2}=A_{h}^{\prime \prime}(1)+\lambda_{h}-\lambda_{h}^{2} . \quad \lambda_{i 2}(i=1,2, \ldots, N)$ is the arrival rate of the low priority service for each site. The generation function, mean value and variance of each station for low priority service arrival process are $A(z), \lambda_{i 2}(i=1,2, \ldots, N)=\lambda=A^{\prime}(1)$ and $\sigma_{\lambda}^{2}=A^{\prime \prime}(1)+\lambda-\lambda^{2}$. The low priority queues of the site $i(i=1,2, \ldots, N)$ obtain the transmission right at time $t_{n}$ and send the information packets arriving before time $t_{n}$ by the gated service rule, the transmission service time of an information packet in the queue follows the identical independent probability distribution with the generation function, mean value and variance are $B(z), \beta=B^{\prime}(1)$ and $\sigma_{\beta}^{2}=B^{\prime}(1)+\beta-\beta^{2}$. When the site $i$ completes the low-priority service information packet transmission before the station time $t_{n}$ arrives, after a switch time $\gamma$ [its generation function, the mean value and variance are $R(z), \gamma=R^{\prime}(1)$ and $\sigma_{\beta h}^{2}=B_{h}^{\prime \prime}(1)+\beta_{h^{-}}$$\left.\beta_{h}^{2}\right], \mathrm{AP}(\mathrm{BS}$ or Master) through the multi-point scheduling method to check whether the high priority queue is empty, and query $i+1$ site. If the high priority service queue is not empty, the high priority service of each station is unified according to the exhaustive service rule at time $t_{n}^{*}$ to send information packets, and the generation function, the mean value and variance are $B_{h}(z), \beta_{h}=B_{h}^{\prime}(1)$ and $\sigma_{\beta h}^{2}=B_{h}^{\prime \prime}(1)+\beta_{h}-\beta_{h}^{2}$. After the high priority business of each station is empty, the $i+1$ site starts the low priority service transmission at time $t_{n+1}$. The transmission of the high priority service and the low priority service of each site are alternately accepted service by the AP (BS or Master) and are accessed according to the rules of the exhaustive service and the gated service respectively.

At the time of $t_{n}, t_{n}^{*}$ and $t_{n+1}\left(t_{n}<t_{n}^{*}<t_{n+1}\right)$, the 
whole system state can be expressed by the variables $\left\{\xi_{1}(n), \xi_{2}(n), \ldots, \xi_{N}(n), \xi_{h}(n)\right\},\left\{\xi_{1}\left(n^{*}\right), \xi_{2}\left(n^{*}\right), \ldots\right.$, $\left.\xi_{N}\left(n^{*}\right), \xi_{h}\left(n^{*}\right)\right\}$ and $\left\{\xi_{1}(n+1), \xi_{2}(n+1), \ldots, \xi_{N}(n+1)\right.$, $\left.\xi_{h}(n+1)\right\}$. The state of the system can be described by the Markov chain, which is aperiodic and experienced.

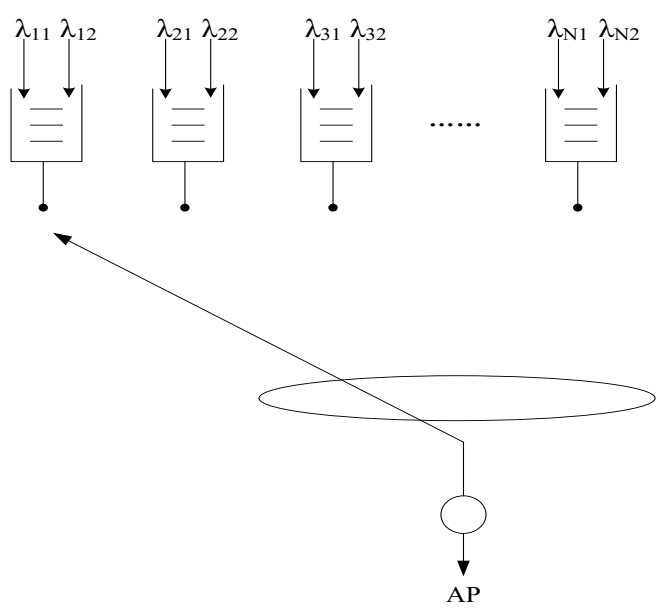

Fig.2. Distinguish the priority MAC protocol system diagram.

\subsection{Protocol model characteristics}

According to the Markov chain theory, under the condition of $\sum_{i=1}^{N} \lambda_{i} \beta_{i}+\lambda_{h} \beta_{h}<1$, the systemgeneration function is:

$G_{i+1}\left(z_{1}, z_{2}, \cdots, z_{N}, z_{h}\right)=$

$R\left[\prod_{j=1}^{N} A_{j}\left(z_{j}\right) A_{h}\left(B_{h}\left(\prod_{j=1}^{N} A_{j}\left(z_{j}\right) F\left(\prod_{j=1}^{N} A_{j}\left(z_{j}\right)\right)\right)\right)\right] \bullet$

$G_{i}\left[z_{1}, z_{2}, \cdots, B_{i}\left(\prod_{j=1}^{N} A_{j}\left(z_{j}\right) A_{h}\left(B_{h}\left(\prod_{j=1}^{N} A_{j}\left(z_{j}\right) F\left(\prod_{j=1}^{N} A_{j}\left(z_{j}\right)\right)\right)\right)\right)\right.$,

$\left.z_{i+1}, \cdots, z_{N}, B_{h}\left(\prod_{j=1}^{N} A_{j}\left(z_{j}\right) F\left(\prod_{j=1}^{N} A_{j}\left(z_{j}\right)\right)\right)\right]$

$$
i=1,2, \ldots, N
$$

Where $F(z)$ is the probability generation function of the transmission time distribution for the information packets arriving in any time slot according to the exhaustive service rule. And it is assumed that the memory capacity of each site is large enough and don't cause loss of information packets.
The average information packets stored is $\mathrm{g}_{i}(j)$ in the $j$ station memory, when the service starts at $t_{n}$.

$$
\begin{gathered}
g_{i}(j)=\lim _{z_{1}, \cdots, z_{N}, z_{h} \rightarrow 1} \frac{\partial G_{i}\left(z_{1}, z_{2}, \cdots, z_{N}, z_{h}\right)}{\partial z_{j}} \\
i=1,2, \ldots, N, h ; j=1,2, \ldots, N, h
\end{gathered}
$$

\subsubsection{Mean queue length}

The derivatives of Eqs (1) and (2) give the mean queue length as:

The mean queue length of the low priority service is:

$$
g_{i}(i)=\frac{N \lambda \gamma}{1-\rho_{h}-N \rho}
$$

The mean queue length of the high priority service is:

$$
g_{i h}(h)=\frac{\lambda_{h} \gamma\left(1-\rho_{h}\right)}{1-\rho_{h}-N \rho}
$$

\subsubsection{The average delay}

The average delay of the information packet is the time from the arrival of the information packet into the site memory until it was sent.

The average delay for each site's low priority business information packet is:

$$
E(w)=\frac{(1+\rho) g_{i}(i, i)}{2 \lambda g_{i}(i)}
$$

The average delay for each site's high priority business information packet is:

$$
E\left(w_{h}\right)=\frac{g_{\text {ih }}(h, h)}{2 \lambda_{h} g_{i h}(h)}-\frac{\left(1-2 \rho_{h}\right) A_{h}^{\prime \prime}(1)}{2 \lambda_{h}^{2}\left(1-\rho_{h}\right)}+\frac{\lambda_{h} B_{h}^{\prime \prime}(1)}{2\left(1-\rho_{h}\right)}(6)
$$

\subsubsection{The average query cycle and throughput}

The average query cycle for $\mathrm{N}$ sites is:

$$
E(\theta)=\frac{N_{\gamma}}{1-\rho_{h}-N \rho}
$$

The throughput of the system is the number of 
packets transmitted by the system per unit of time.

The throughput of the system is:

$$
T=N \rho+\rho_{h}
$$

\subsection{Method of improving QoS guarantee}

Based on the traffic priority of the polling mechanism: in the original IEEE 802.16 MAC, the IP head of the Taxonomy of Service (ToS) field that has 3bit priority sub field mapping into the above four different Qos level, see Table 2. The same level of business uses the same type of scheduling, no longer distinguish priority, here we will further subdivide the rtPS business and nrtPS business to distinguish priority services. "011" of rtPS and "001" of nrtPS in the ToS field are the high priority service identifier in the same service, and "100" of rtPS and "010" of nrtPS are used as low priority service identifier in the same business. In the IEEE 802.16 MAC polling mode, the BS periodically queries the different business queues of the SS site. First, the higher priority ${ }^{[8,9]}$ business queue is authorized to send the information in the exhaustive service mode, that is, it sends the full information packets of the queue and the packets of information to be reached during the transmission, until the class of business queues on the query SS site is empty. When the SS high priority service queue is empty, the BS starts to query the service queue with the lower priority and sends the information according to the policy of the gated service. That is, it sends the information packets that arrive before the transmission start. The information packets arriving during transmission will wait for the next chance to send. In this way, the service of the polling mode in the IEEE 802.16 MAC is distinguished by the different service policies of the exhaustive service and the gated service, which makes it have better flexibility and QoS guarantee.

Table 2. Correspondence table for ToS field and IEEE 802.16 service type

\begin{tabular}{|c|c|c|c|}
\hline $\begin{array}{c}\text { Business } \\
\text { type }\end{array}$ & ToS field & $\begin{array}{c}\text { Scheduling } \\
\text { strategy }\end{array}$ & Business \\
\hline UGS & $111-101$ & $\begin{array}{c}\text { reserve } \\
\text { resources }\end{array}$ & VoIP \\
\hline rtPS & $100-011$ & $\begin{array}{c}\text { real-time } \\
\text { polling }\end{array}$ & $\begin{array}{c}\text { MEPG video } \\
\text { stream }\end{array}$ \\
\hline
\end{tabular}

\begin{tabular}{|c|c|c|c|}
\hline nrtPS & $010-001$ & $\begin{array}{c}\text { non-real-time } \\
\text { polling }\end{array}$ & $\begin{array}{c}\text { interactive } \\
\text { game }\end{array}$ \\
\hline BE & 000 & FIFO & $\begin{array}{c}\text { WWW E- } \\
\text { mail }\end{array}$ \\
\hline
\end{tabular}

\section{Analysis and evaluation}

Based on the Markov chain and the probability generation function of the wireless computer network MAC protocol model, combined with the characteristics of IEEE 802.16, the length of a slot is defined as 200 microseconds, the uplink TDMA frame length is 0.01 seconds, that is, Contains 50 time slots. SS station number is set to 50 , through the theoretical calculation and simulation experiments, the average information packets stored and the average delay are as follows.

Table 3. Average information packets stored

\begin{tabular}{|c|c|c|c|c|}
\hline $\begin{array}{c}\lambda=\lambda_{\mathrm{h}} \\
\text { Inform } \\
\text { ation } \\
\text { packets } \\
\text { Time } \\
\text { slot) }\end{array}$ & $\begin{array}{c}\text { High } \\
\text { priority } \\
\text { business } \\
\text { theoretica } \\
\text { l value }\end{array}$ & $\begin{array}{c}\text { High } \\
\text { priority } \\
\text { business } \\
\text { experime } \\
\text { ntal } \\
\text { value }\end{array}$ & $\begin{array}{c}\text { Low } \\
\text { priority } \\
\text { business } \\
\text { theoretical } \\
\text { value }\end{array}$ & $\begin{array}{c}\text { Low } \\
\text { priority } \\
\text { business } \\
\text { experime } \\
\text { ntal } \\
\text { value }\end{array}$ \\
\hline 0.00050 & 0.00057 & 0.00057 & 0.02865 & 0.02863 \\
\hline 0.00100 & 0.00134 & 0.00132 & 0.06711 & 0.06715 \\
\hline 0.00150 & 0.00241 & 0.00242 & 0.12146 & 0.12172 \\
\hline 0.00200 & 0.00404 & 0.00411 & 0.20408 & 0.20461 \\
\hline 0.00250 & 0.00405 & 0.00404 & 0.34014 & 0.34065 \\
\hline 0.00300 & 0.01257 & 0.01269 & 0.63829 & 0.63714 \\
\hline
\end{tabular}

Table 4. Information packets average delay

\begin{tabular}{|c|c|c|c|c|}
\hline $\begin{array}{c}\lambda=\lambda_{\mathrm{h}} \\
\text { (Inform } \\
\text { ation } \\
\text { packets } \\
\text { Time } \\
\text { slot) }\end{array}$ & $\begin{array}{c}\text { High } \\
\text { priority } \\
\text { business } \\
\text { theoretic } \\
\text { al value }\end{array}$ & $\begin{array}{c}\text { High } \\
\text { priority } \\
\text { business } \\
\text { experim } \\
\text { ental } \\
\text { value }\end{array}$ & $\begin{array}{c}\text { Low } \\
\text { priority } \\
\text { business } \\
\text { theoretical } \\
\text { value }\end{array}$ & $\begin{array}{c}\text { pow } \\
\text { priority } \\
\text { business } \\
\text { experiment } \\
\text { al value }\end{array}$ \\
\hline 0.00050 & 0.39131 & 0.39624 & 28.59122 & 28.61720 \\
\hline 0.00100 & 0.80956 & 0.79581 & 34.08481 & 34.06318 \\
\hline 0.00150 & 1.27151 & 1.27191 & 41.84967 & 41.90759 \\
\hline 0.00200 & 1.81122 & 1.80871 & 53.65867 & 53.65264 \\
\hline 0.00250 & 2.47724 & 2.50196 & 72.71238 & 72.65779 \\
\hline 0.00300 & 3.63032 & 3.64774 & 115.73909 & 115.60939 \\
\hline
\end{tabular}


Table 5. Average query cycle and throughput

\begin{tabular}{|c|c|c|c|c|}
\hline $\begin{array}{c}\lambda=\lambda_{\mathrm{h}} \\
\text { Inform } \\
\text { ation } \\
\text { packets } \\
\text { / Time } \\
\text { slot) }\end{array}$ & $\begin{array}{c}\text { Query } \\
\text { cycle } \\
\text { theoretical } \\
\text { value }\end{array}$ & $\begin{array}{c}\text { Query } \\
\text { cycle } \\
\text { experime } \\
\text { ntal } \\
\text { value }\end{array}$ & $\begin{array}{c}\text { Through } \\
\text { put } \\
\text { theoretic } \\
\text { al value }\end{array}$ & $\begin{array}{c}\text { Throughp } \\
\text { ut } \\
\text { experime } \\
\text { ntal value }\end{array}$ \\
\hline 0.00050 & 57.30660 & 57.29980 & 0.12750 & 0.12490 \\
\hline 0.00100 & 67.11409 & 67.11825 & 0.25500 & 0.25014 \\
\hline 0.00150 & 80.97166 & 80.91685 & 0.38250 & 0.37551 \\
\hline 0.00200 & 102.04082 & $\begin{array}{c}102.1795 \\
0\end{array}$ & 0.51000 & 0.50062 \\
\hline 0.00250 & 136.05442 & $\begin{array}{c}136.1732 \\
0\end{array}$ & 0.63250 & 0.62541 \\
\hline 0.00300 & 212.76596 & $\begin{array}{c}212.4554 \\
0\end{array}$ & 0.76500 & 0.74973 \\
\hline
\end{tabular}

Table 3 to table 5 shows that the improved protocol theoretical analysis is consistent with the experimental results, through the IEEE 802.16 MAC protocol IP head ToS sub field settings, providing services to the SS site in business priority. Table 3 shows that the average information packets increases with the increase of the arrival rate, the information packet arrival rate is from 0.0005 to 0.003 , and the information packets stored in the high priority service and the low priority service is increased by more than 20 times, but the distinction between high-priority and low-priority services remains clear. Table 4 shows the same rule on the average delay of the information packet. High-priority and low-priority services are clearly differentiated as the arrival rate and system load increase. Table 5 verifies the consistency between theoretical and experimental analys is of query cycles and throughput.

\section{Conclusions}

This paper based on the four original types of service scheduling makes some improvements in wireless metropolitan area network IEEE 802.16 MAC protocol, and further provides polling scheduling strategies based on service priority. Then, the theoretical value of the average number of information packets and the average delay of the information packet is compared with the experimental value, in the case of high priority business and low priority business. And the theoretical value and the experimental value of the query cycle and throughput are basically consistent. So, they are proved that the improved scheduling strategy improves the flexibility of protocol scheduling and better guarantees the QoS of different service flows. Compared with the traditional wired network, although the existing wireless network service quality and performance there are still some gaps. However, with the popularity of wireless networks and the development of a variety of business applications, the research and improvement of MAC layer QoS mechanism in wireless metropolitan area network has clear practical significance and application value. In the future work, we will continue to study the priority of the polling service strategies, the use of new hybrid services, such as gatedexhaustive service, gated-gated service, exhaustiveexhaustive service to guarantee the service quality of data service, and improve the utilization rate of system resources.

\section{Acknowledgements}

This work was supported by the National Natural Science Foundation of China under Grant Nos.61461054 and 61461053 .

\section{References}

1. YaNan Sun, ZhongQiang Pan, Effective Bandwidth Allocation Mechanism of Supporting QoS in IEEE 802.16 Standard, Computer and Modernization, vol.9,2529,(2016).

2. Jun Yu, GuangWei Bai, Hang Shen, DiffServ-based congestion control mechanism in IEEE 802.16 Mesh networks, Application Research of Computers, vol 31,no.9,(2014).

3. ZhiJun Yang, HongWei Ding, Zheng Guan, LongZheng Zhu, A New Transportation Control System Based On Priority Differentiated Polling Strategy, Proceedings of 2015 World Conference on Control, Electronics and Electrical Engineering,vol.116,38-44,(2015).

4. ZhiJun Yang, HongWei Ding, Characteristics of a TwoClass Polling SystemModel, TSINGHUA SCIENCE AND TECHNOLOGY, vol.19,no.5,516-520,(2014).

5. ZhiJun Yang, HongWei Ding, ChanLong Chen, Research on E(x) Characteristics of Two-Class Polling System of Exhaustive-Gated Service, Acta Electronica 
Sinica, no.4,775-778,(2014).

6. Jia Guo, JiaLong Xiong, ZhiJun Yang, HongWei Ding, The analysis of performance for a priority distinction double-queue and single-server communication network, International Conference on Control Engineering and Electronics Engineering, Vol. 95,(2014).

7. JiaLong Xiong, ZhiJun Yang, HongWei Ding, MengYao Wang, The Analysis of Performance for Priority Distinction Double-queue and Triple-server Communication Network, 2015 International Conference on Computer Science and Software Engineering,810826,(2015).

8. Zheng Guan, Zhijun Yang, Wenhua Qian, Min He, Research Article On Two-Level State-Dependent Routing Polling Systems with Mixed Service, Mathematical Problems in Engineering,1-9,(2015).

9. Min He, Zheng Guan, Liyong Bao, An Analysis of Polling Access Protocol for Wireless Sensor Networking, The 5th Annual IEEE International Conference on Cyber Technology in Automation, Control and Intelligent Systems,720-724,(2015). 